Egyptian

Orthodontic Journal

\title{
A NEWLY CONSTRUCTED COMPUTER PROGRAM FOR THREE DIMENSIONAL ANALYSIS OF TOOTH MOVEMENT
}

\author{
Khaled M. Fawzy ${ }^{*}$ Noha E. Sabet ${ }^{* *}$ \\ Ibrahim M. Negm ${ }^{* * *}$
}

ABSTRACT

The aim of this study went along two parallel ways; the first was to develop a new method for three dimensional 3D imagining of the dental cast, and the second was to evaluate it's accuracy in analyzing the different tooth movements presented by three different palatal expanders, (A) Nitanium Palatal Expander 2 (NNPE2), (B) Compound Palatal Bar (CPB) and (C) Quad Helix (QH).Thirty healthy Egyptian girls each exhibiting unilateral or bilateral posterior crossbite, and required palatal expansion as part of their comprehensive orthodontic treatment were selected and then they were equally divided into one of three groups in a random fashion. These groups were classified according to the type of expander utilized: Group A: Ten girls utilizing the Nitanium Palatal Expander 2 (NNPE 2), Group B: Ten girls utilizing the Compound Palatal Bar (CPB), Group C: Ten girls utilizing the Quad Helix $(\mathrm{QH})$. Each subject was clinically examined, and an orthodontic diagnostic study cast was recorded. Both extraoral and intraoral photography were taken. In addition to panoramic and occlusal radiographs, Lateral and posteroanterior cephalometric

* Professor of Orthodontic. Orthodontic and Pediatric Dentistry Departmernt, Faculty of Dentistry, Ain Shams University.

** Associate Professor of Orthodontic. Orthodontic and Pediatric Dentistry Department, Faculty of Dentistry, Ain Shams University.

${ }^{* * *}$ Assistant Lecturer of Orthodontic. Orthodontic and Pediatric Dentistry Department, Faculty of Dentistry, Ain Shams University. 
Egyptian

Orthodontic Journal

radiographs were obtained. A 3D computer program was specially designed for more accurate evaluation of the dental effects induced by the three types of maxillary expanders, For the rotation and extrusion: ANOV $\mathcal{A}$ test showed that there was a statistically significant difference between the three expanders. CPPB expander showed the statistically significant lowest mean followed by $\mathcal{N}$ PE2. QH expander showed the statistically significantly

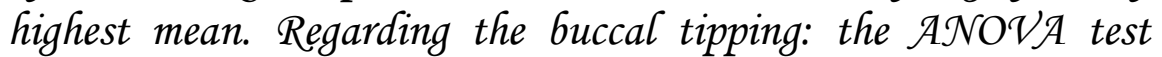
showed that; there was a statistically significant difference between the three expanders. There was no statistically significant difference between (NPPE2) and (CPB) expanders which showed the statistically significantly lowest means. QH expander showed the statistically significantly highest mean. It was concluded that: One of the imperative outcomes exclusive to this study was the development of a new method for 3D imaging of dental casts other than $C \mathcal{T}$ and laser scanning. The reliability of generating $3 D$ dental images using photographic imaging of the dental casts for 3D tooth movement analysis has a great research potential in orthodontics because of its ability to yield accurate and reproducible data. An accurate and comprehensive description of the orthodontic tooth movements in various clinical situations is best assessed three dimensionally. The $\mathcal{N} P \mathrm{PE}^{2}, \mathrm{CPB}$, and $\mathrm{QH}$ expanders are capable of expanding the maxillary dentition and alveolar process and are equally capable of correcting posterior crossbite.

\section{INTRODUCTION}

Recent advances in the application of three-dimensional (3D) imaging for dental purposes have made possible a more accurate assessment of tooth positional changes. ${ }^{(1,2)}$ Laser scanners are now widely used in orthodontics and maxillofacial surgery for the $3 \mathrm{D}$ acquisition of dental casts or the face. ${ }^{(1,3)}$ 
The reliability of generating 3D dental images using surface laser scanners has been investigated, and those devices were reported to have great research potential in orthodontics because of their ability to yield accurate and reproducible data. Differences between direct measurements made on dental casts and those made on computer-reconstructed images generated by surface laser scanners have shown that those devices were highly accurate for dental cast analysis. ${ }^{(4,5)}$

The cephalometric assessment and direct cast analysis alone could not provide an accurate and comprehensive description of the orthodontic tooth movements that occurred in maxillary expansion clinical situations. As the actual treatment results were 3D in nature, cephalometric and direct cast analysis worked in two-dimensional 2D, which meant that the clinical changes were only reported as a 2D projection of a 3D movement in six degrees of freedom, therefore leading to loss in information.

In 2003 Virgilio et. al., ${ }^{(6)}$ used a three-dimensional (3D) electromagnetic instrument to analyze the six-month effects of a nickel-titanium (NiTi) palatal expander on the dental and palatal structures of four primary (mean age 5.8 years) and nine mixed dentition children (mean age 8.7 years), with a posterior unilateral cross bite.

Standardized dental and palatal landmarks were digitized; the collected data were analyzed with geometric-mathematical models. During a six-month interval, the natural growth and development of the dental arches and hard tissue palate was negligible, as assessed in seven control children (two in the primary dentition, mean age 4.4 years; five in the mixed dentition, mean age 7.7 years).

In all children the cross bite was completely corrected. Dental expansion was more than or corresponded to the palatal expansion. A smoothing of the size-independent (shape) palatal curvature in the transverse plane was observed. No differences in maximum palatal height were noted. Symmetrical derotation of the anchorage teeth in a distal direction occurred in almost all children. The inclination of the facial axis of the clinical crown (FACC) in the anatomical transverse plane of those teeth with differences between dental and palatal expansion showed significant modifications. The clinical crown height of anchorage teeth remained nearly the same in all patients. No significant modifications in the mandibular arch size were observed.

They concluded that the increase in maxillary arch width, especially in younger children, was probably due to a combination of different effects: opening of the midpalatal suture, tipping of the alveolar process and molar tipping.

Nanci L. Oliveira et al., $(2004)^{(7)}$ used a 3-dimensional surface laser scanning technique and computerized cast analysis, in addition to analysis of 
anteroposterior cephalograms, to assess the morphologic changes of the palate by 2 kinds of expanders: tissue borne (Haas) and tooth borne (Hyrax). Cast analysis demonstrated that, although all patients started treatment with similar malocclusion, treatment outcomes were different depending on the appliance used. Both appliances generated maxillary expansion.

However, the appliances performed differently to achieve the final expansion. Haas appliances demonstrated a greater orthopedic movement (i.e., improvement of the mean interpalatal distance) and Hyrax appliances demonstrated dentoalveolar expansion by increasing the mean palatal angulation of the alveolus. Anteroposterior cephalometric analysis showed that both appliances increased mean maxillary width and mean intermolar distance significantly.

In 2007 Podesser B. et. al. ${ }^{(8)}$, evaluated the effects of rapid maxillary expansion in growing children using computer tomography scanning. The changes were evaluated on pre- and post-treatment computer tomographic scans taken using a low-dosage protocol. Their results demonstrated a clear applianceinduced effect in all patients, although the relative contribution of dental, alveolar, and skeletal changes varied from one subject to the other. The average expansion, measured at the molar crowns, was $3.6 \mathrm{~mm}$, whereas the actual sutural opening, the main aim of RME, was as low as $1.6 \mathrm{~mm}$.

Marini I. et. al. $2007^{(9)}$, assessed the dimensional changes before and after rapid maxillary expansion (RME) by a digital photogrammetric technique. The transverse diameters and volumetric variations of the palate were measured by photogrammetry on study casts taken at three different phases of therapy: at the beginning of treatment (T1), on removal of the rapid expander, after expansion and retention for three months (T2), and six months after appliance removal (T3). Their findings demonstrated a significant relapse $(\mathrm{P}<0.001)$ in the dental transverse diameter in all patients six months after appliance removal, although the palatal volume remained stable.

So the aim of this study went along two parallel ways; the first was to develop a new method for three dimensional 3D imagining of the dental cast, and the second was to evaluate its accuracy in analyzing the different tooth movements presented by three different palatal expanders, (A) Nitanium Palatal Expander 2 (NPE2) , (B) Compound Palatal Bar (CPB) and (C) Quad Helix (QH) so the 3D cast imaging acquisition was done by photographic imaging for the dental casts from four standardized predefined positions. Those images were then fed to a custom made 3D computer software program that provided a 3D analysis of tooth movements after maxillary expansion utilizing the three different expanders. 
Egyptian

Orthodontic Journal

\section{Material \& Methods}

This study consisted of thirty healthy Egyptian girls each exhibiting unilateral or bilateral posterior crossbite, and required palatal expansion as part of their comprehensive orthodontic treatment. The subjects were selected and treated at the out patient clinic of the Orthodontic Department, Faculty of Dentistry, Ain Shams University.

The criteria considered for subject selection in this study was as follow:

- All subjects were in the early permanent dentition stage.

- All subjects had either unilateral or bilateral posterior crossbite involving at least the first permanent molar.

- All subjects had normal mandible.

- All subjects did not perform any previous orthodontic treatment.

- All subjects were free of any systemic disease affecting craniofacial growth, previous periodontal disease, and dental pathology.

The subjects were equally divided into one of three groups in a random fashion. These groups were classified according to the type of expander utilized:

- Group A: Ten girls utilizing the Nitanium Palatal Expander 2 (NPE 2) whose mean age was 13.3 years.

- Group B: Ten girls utilizing the Compound Palatal Bar (CPB) whose mean age was 14.2 years.

- Group C: Ten girls utilizing the Quad Helix $(\mathrm{QH})$ whose mean age was 13.7 years.

The Pretreatment, Post-expansion, \& Post retention Database Records:

Each subject was clinically examined, and an orthodontic diagnostic study cast was recorded. Both extraoral and intraoral photography were taken. In addition to panoramic and occlusal radiographs, lateral and posteroanterior cephalometric radiographs were obtained.

All of the above mentioned records were taken for each subject before treatment (T1), after expansion treatment (T2) except for panoramic and occlusal radiographs, and after a three month retention period (T3) except for occlusal radiographs. 
Before the study, all the subjects' parents gave their informed consent after receiving a full explanation of the aim and design of this study for the additional radiographic records required. The study protocol was approved by the local ethics committee.

\section{Different types of maxillary expanders used (A) Nitanium Palatal Expander 2 (NPE2)*}

\section{Expander Design \& Properties:}

The NPE 2 incorporated (a) 0.036" NiTi bar with a central loop, (b) an innovative lingual attachment, (c) 0.036 " Ortholoy arms, and (d) molar loops for unilateral and bilateral adjustments Fig 1. A locking indentation in the lingual attachment fastened the appliance securely to the maxillary molar band. To prevent removal or accidental dislodging, the appliance was also tied in with ligatures.

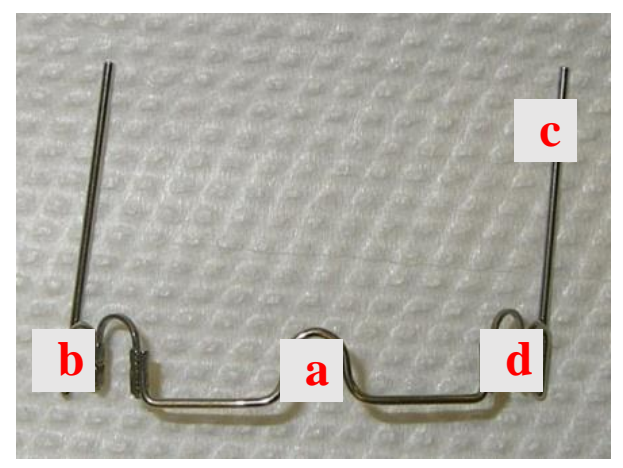

Fig (1) Nitanium Palatal Expander 2 (NPE2)

\section{(B) Compound Palatal Bar SE (CPB) $)^{* *}$}

\section{Expander Design \& properties:}

The CPB incorporated (a) 0.036" St.St. bar with a central loop, (b) lingual attachment, (c) 0.036" St.St. arms, and (d) lateral molar loops for unilateral and bilateral adjustments. In addition to (e) two super elastic NiTi loops Fig 2. A locking indentation in the lingual attachment fastened the appliance securely to the maxillary molar band. To prevent removal or accidental dislodging, the appliance was also tied in with ligatures.

* Ortho Organizers, INC, USA

${ }^{* *}$ Forestadent, Germany 


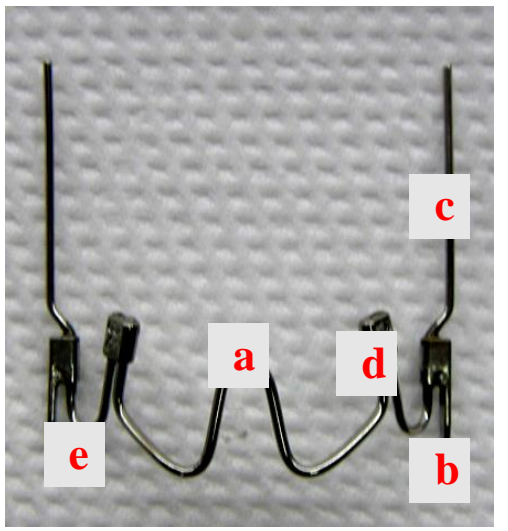

Fig (2) Compound Palatal Bar (CPB)

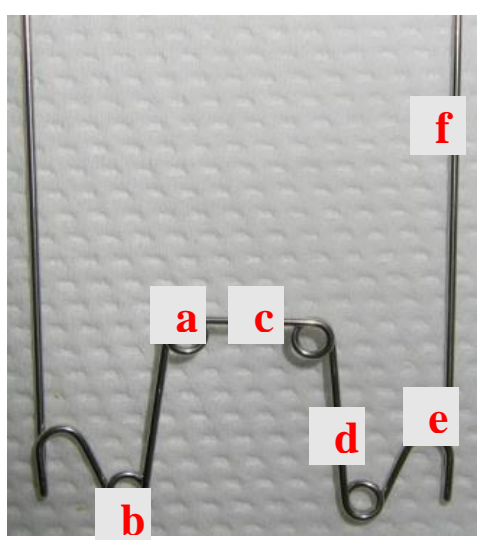

Fig (3) Quad Helix

\section{(C) Quad Helix (QH)}

\section{Expander Design \& Properties:}

The QH was prefabricated from a $0.036 "$ St.St. wire. It incorporated four helices, (a) two anterior and (b) two posterior. It also incorporated (c) an anterior bridge, (d) two inner lateral arms, (e) lingual attachments, and (f) two outer extension arms for unilateral and bilateral adjustments. A locking indentation in the lingual attachment fastened the appliance securely to the maxillary molar band. To prevent removal or accidental dislodging, the appliance was also tied in with ligatures.

\section{Three dimensional analysis $\quad(3 D)$}

A 3D computer program was specially designed for more accurate evaluation of the dental effects induced by the three types of maxillary expanders (NPE2, CPB, and $\mathrm{QH})$. The computer program was customized with the consideration that it should be superior or equal in accuracy to the standard methods. In order to perform a 3D analysis of our pretreatment (T1) postexpansion (T2), and postretention (T3) cases the following data was gathered; A 3D vector data for the study cast digitization, where each point of interest was defined by its $\mathrm{x}, \mathrm{y}, \mathrm{z}$ coordinates in space. In addition to a superimposition plane (three points) to which the dental changes could be relatively compared.

\section{Steps for the 3D computer program construction}

The 3D data was assembled by digitizing multiple images of each cast. In order to feel the depth factor, which gives the 3D effects; our viewing system 
(the eyes) draws a perspective projection of any image onto the retina. The further objects appear to be the smaller they become. Two parallel lines would appear to be a single point as they vanish in the horizon.

Mathematically, that means that every point in the original geometry was multiplied by a certain matrix to produce a new perspective correct point, and vice versa. These points should be projected on a viewing plane, which was $2 \mathrm{D}$ in nature (image plane, computer monitor) as a set of 2D points. Fig 4 (a \& b).
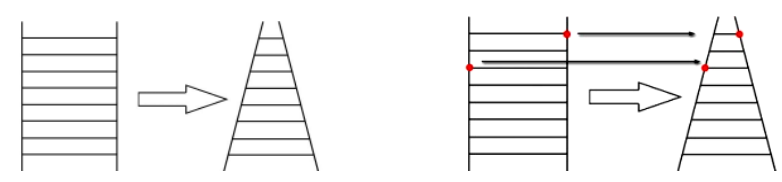

Fig 4(a) \& (b) A schematic diagram representing a perspective image projection

In order to reproduce the original geometry of a study cast from a 2D projected perspective correct image the image had to be unprojected. Fig 5 (a \& b).

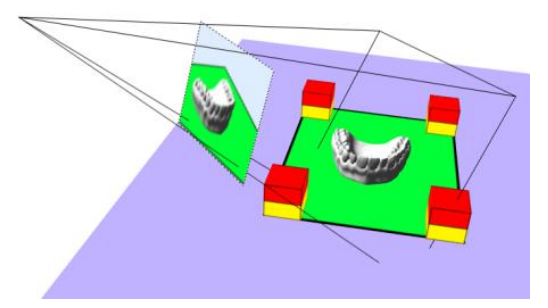

Fig 5(a) 2D study cast image with a projected correct perspective.

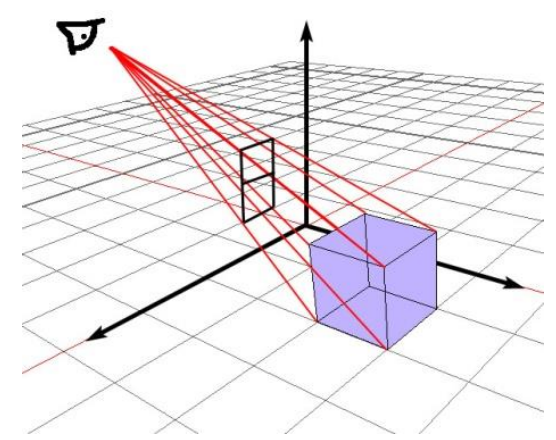

Fig 5(b) Original geometry reproducibility via unprojection. 
In order to unproject the image the original matrices used to produce the image were reconstructed. Those matrices were in direct relation with the camera optics, and the position and orientation relative to the study cast. The used approach for assembling the 3D data was development of a new flexible technique in a trial to simplify the method of 3D acquisition. The currently available methods are either laser or CT scanning which are expensive, complicated, and not readily available. Therefore, the development of a new flexible technique in which relaxation of all parameters was attempted for image acquisition in a $3 \mathrm{D}$ perspective

The matrices elements were deduced using multiple images, from multiple directions Fig (6). View calibration was done by identifying some fixed points on all the images Fig (7).

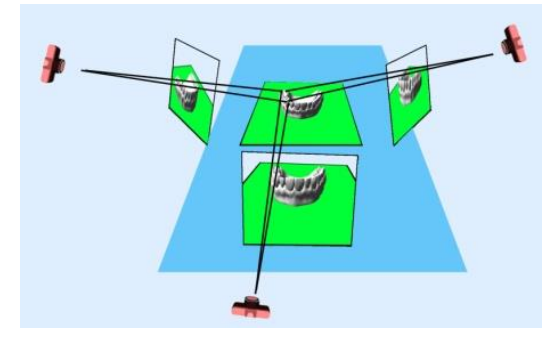

Fig (6) A Diagram for multiple images from multiple directions.

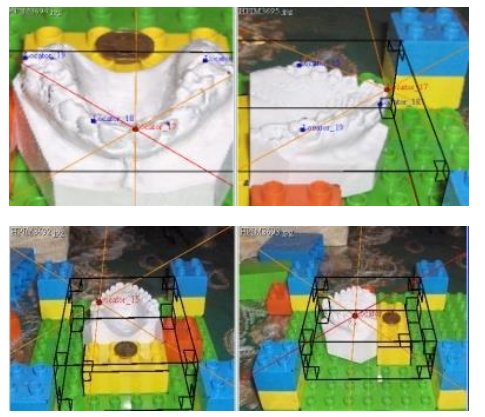

Fig (7) A view of calibration by fixed point identification.

To facilitate the calibration process predefined $90^{\circ}$ angles were used during photographic acquisition. Identifying $90^{\circ}$ angles greatly increased the accuracy of the process. Fig (8). 
Egyptian

Orthodontic Journal

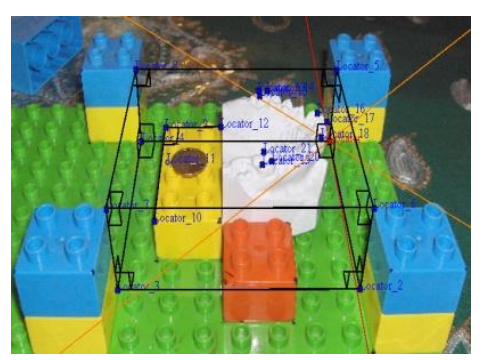

Fig (8) Predefined $90^{\circ}$ angles

After calibration image-modeler computer software program ${ }^{1}$ was used for the process to provide the necessary $3 \mathrm{D}$ coordinates of the required points.

A program was specially developed to use those $3 \mathrm{D}$ coordinates to reproduce a 3D triangle, representing the maxillary first permanent molars, in the right positions and orientation, with vertices at the following points: Fig (9)

Disto-Buccal cusp

Mesio-Buccal cusp

Mesio-Palatal cusp.

The program also used the $3 \mathrm{D}$ coordinates to reproduce another $3 \mathrm{D}$ triangle representing the anterior superimposition plane, with vertices at the following points: Fig (9)

- Mesial contact point of U1

- Mid point of incisal edge of U2 bilaterally

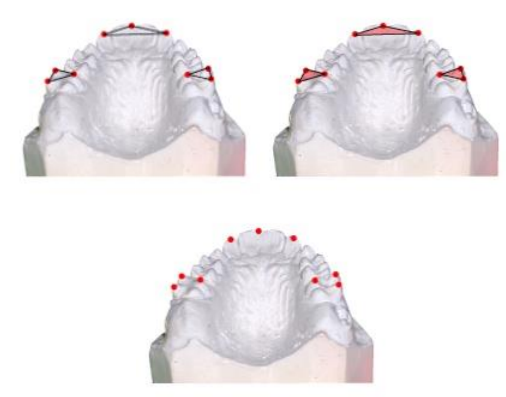

Fig (9) Vertices, 3D coordinates, 3D triangles

\footnotetext{
${ }^{1}$ Cycore REALVIZ ImageModeler technology, San Mateo, Calif. and Sophia Antipolis, France.
} 
An avatar (a generic 3D molar model representing the maxillary first permanent molar, was scaled and oriented with the triangle, to give a visual clue to the 3D movements at T1,T2, and T3.Fig(10)
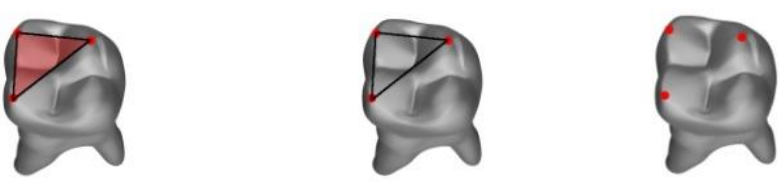

Fig (10) A scaled avatar (a generic 3D molar model)

To insure proper reporting of dimensions, a known measure was fed to the program to map the virtual world space units to real world space units. Fig (11)

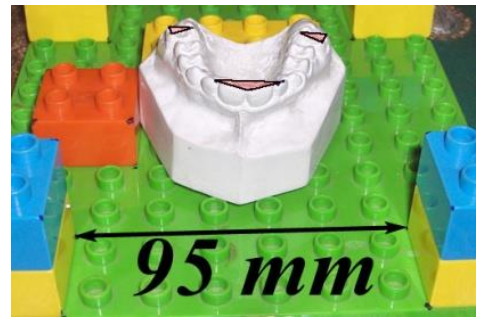

Fig (11) known measure fed to the program

The program calculated the reference planes, and then automatically superimposed the pretreatment and postexpansion study casts on the unified plane. The results were displayed as a 3D interactive scene. Fig (12)

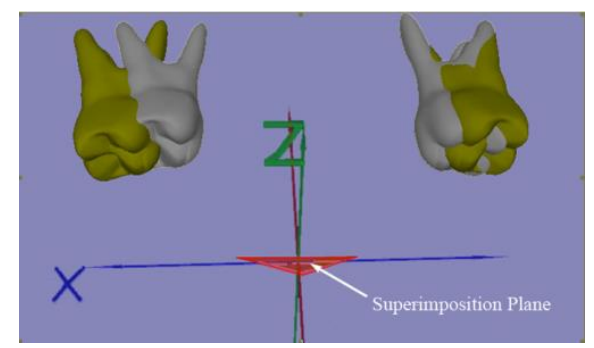

Fig (12) Superimposition of pretreatment and post expansion study casts on the unified plane

The resulting 3D scene showed the real 6 degrees of freedom movements for the molar, produced by each expander. 
The system adopted in this study had an error $1 / 100$ of a millimeter. That was detected by measuring a known distance between two specific selected points which was $95 \mathrm{~mm}$., then comparing it to the same measurement as reported by the system which was $94.98 \mathrm{~mm}$, with an error of $0.02 \mathrm{~mm}$. Fig (13)

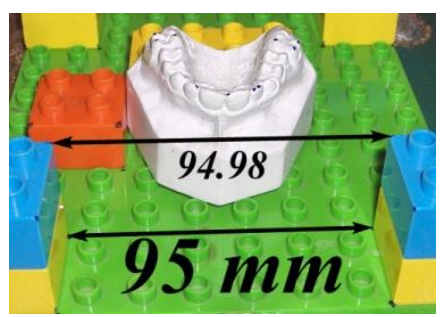

Fig (13 ) Detection of system error.

\section{Evaluation of treatment results in 3D:}

All measures were performed on two levels: Individual projections of each point on the 3 coordinate axes.

- The center of mass for occlusal triangle.

The center of mass for the occlusal triangle gave an estimate of the overall dental movements, tipping, translation, and rotation. The projection of individual points gave the exact movements for the point in each axis (x, y, and z). Fig (14)
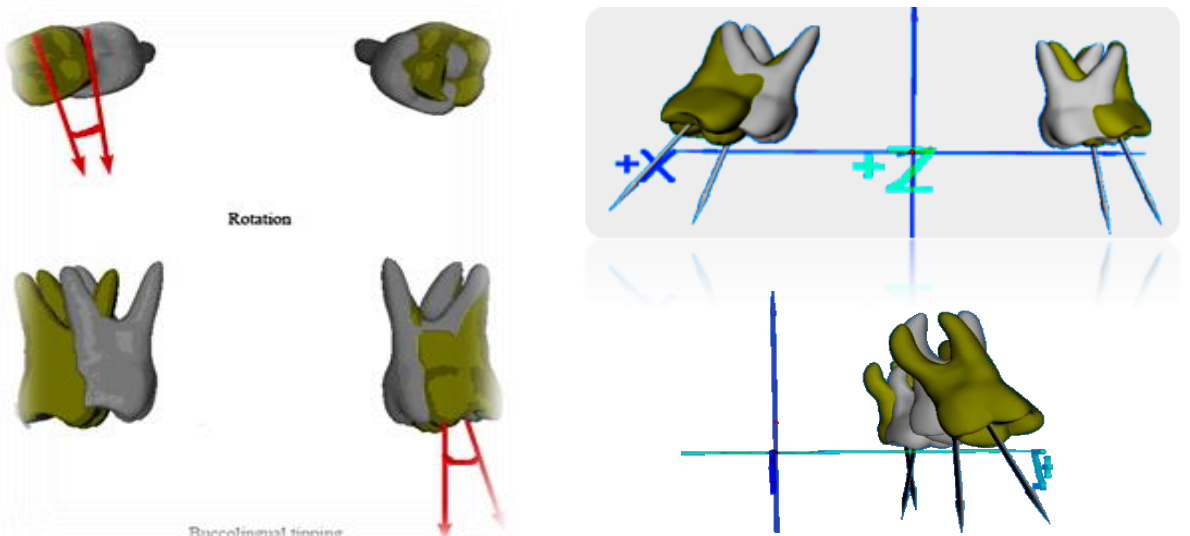

Fig(14) Evaluation of rotation \& buccal tipping in degrees 


\section{Statistical analysis:}

Data were presented as means and standard deviation values. One-way ANOVA (Analysis of Variance) was used to compare between means of the three groups. Duncan's post-hoc test was used for pair-wise comparison between the means when ANOVA test was significant

\section{RESULTS}

The means, standard deviation (SD), standard error (SE) values for rotation, extrusion and buccal tipping of the maxillary first molars that occurred with the three expenders used in this study. Results of ANOVA and Duncan's tests are presented in the following table (1) and figure 16 (a \& b):

Table 1: The means, standard deviation (SD), standard error (SE), ANOVA and Duncan's tests for rotation, extrusion and buccal tipping of the maxillary first molars for the three expanders.

\begin{tabular}{|c|c|c|c|c|c|c|c|c|c|c|c|}
\hline \multirow{2}{*}{ Movement } & \multicolumn{3}{|c|}{$\begin{array}{l}\text { NPE2 } \\
(n=5)\end{array}$} & \multicolumn{3}{|c|}{$\begin{array}{c}\text { CPB } \\
(n=5)\end{array}$} & \multicolumn{3}{|c|}{$\begin{array}{c}\text { QH } \\
(n=5)\end{array}$} & \multirow{2}{*}{$f$-value } & \multirow{2}{*}{$P$-value } \\
\hline & Mean & SD & SE & Mean & SD & SE & Mean & SD & SE & & \\
\hline Rotation & $13.6^{\mathrm{b}}$ & 1.8 & 0.8 & $11.2^{\mathrm{a}}$ & 1.6 & 0.7 & $25.8^{\mathrm{c}}$ & 1.3 & 0.6 & 119.403 & $<0.001 *$ \\
\hline Extrusion & $1.7^{\mathrm{b}}$ & 0.3 & 0.1 & $1^{\mathrm{a}}$ & 0.3 & 0.1 & $2.7^{\mathrm{c}}$ & 0.6 & 0.2 & 20.776 & $<0.001 *$ \\
\hline Buccal tipping & $4.8^{\mathrm{a}}$ & 0.8 & 0.4 & $3^{a}$ & 1 & 0.4 & $22^{b}$ & 1.9 & 0.8 & 317.346 & $<0.001 *$ \\
\hline
\end{tabular}

\section{Rotation and Extrusion:}

ANOVA test showed that there was a statistically significant difference between the three expanders. CPB expander showed the statistically significant lowest mean followed by NPE2. QH expander showed the highest statistically significant mean.

\section{Buccal tipping:}

ANOVA test showed that there was a statistically significant difference between the three expanders. There was no statistically significant difference between (NPE2) and (CPB) expanders which showed the lowest statistically significant means. QH expander showed the highest statistically significant mean. 


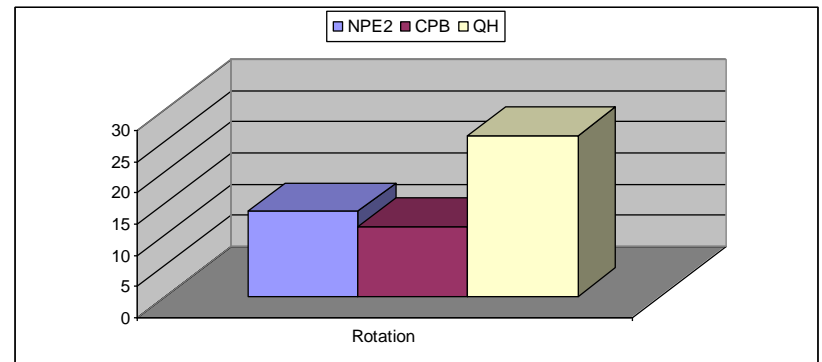

Fig 16 (a) Mean Rotation measurements of the three groups

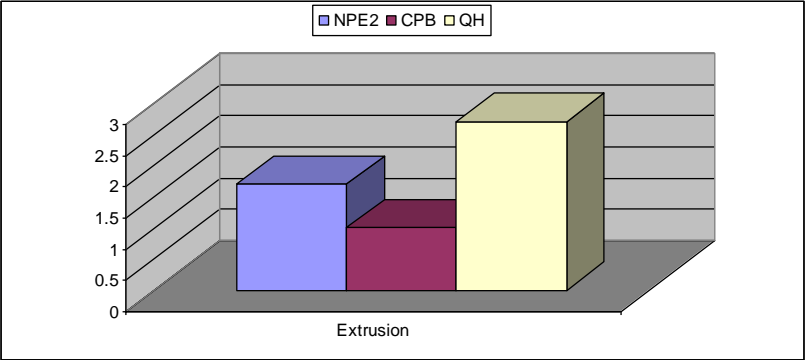

Fig 16 (b) Mean Extrusion measurements of the three groups

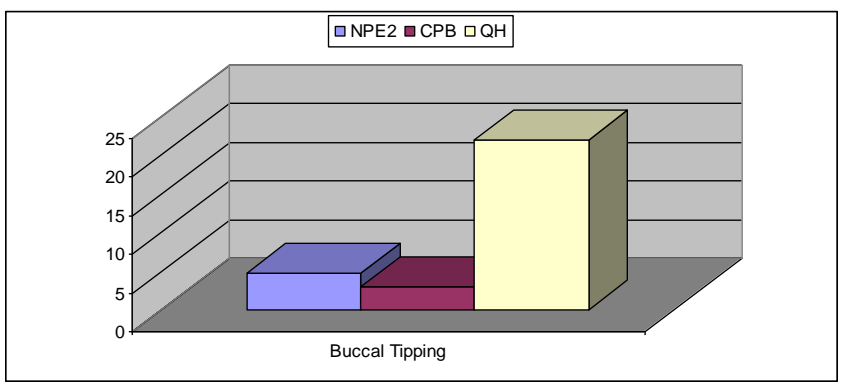

Fig 16 (c) Mean Buccal Tipping measurements of the three groups

\section{DISCUSSION}

This oeuvre was dedicated to develop a new method for three dimensional 3D imagining of the dental cast, and to evaluate its accuracy in analyzing the different tooth movements presented by three different palatal expanders; 
(A) Nitanium Palatal Expander 2 (NPE2), (B) Compound Palatal Bar (CPB) and $(\mathrm{C})$ Quad Helix $(\mathrm{QH})$. Thus the 3D cast imaging acquisition was done by photographic imaging for the dental casts from four standardized predefined positions. Those images were then fed to a custom made 3D computer software program that provided a $3 \mathrm{D}$ analysis of tooth movements after slow maxillary expansion utilizing the three different expanders.

A finding of vast value was the statistically significant difference involving the three expanders as regard to rotational movements of the maxillary first molars. The CPB expander demonstrated the least amount of rotation followed by NPE2 whereas, QH expander had the highest degree of rotation, table (1) and fig (16-a).

Those results are synchronized with those found by Marzban and Nanda, ${ }^{(10)}$ Ciambotti et al, ${ }^{(11)}$ and Sander et. al. ${ }^{(12)}$ The underlying principle might be the deflection force and material stiffness. The deflection force of CPB was previously tested by Sander et al ${ }^{(12)}$. to be $0.5 \mathrm{~N}$ per $\mathrm{mm}$. while that of the QH $3 \mathrm{~N}$ per $\mathrm{mm}$. CPB expander showed notably lowest amount of extrusion trailed by NPE2. QH expander provided drastically the highest extrusion, table (1) and fig (16-b). The rational explanation could be that CPB produced the lowest forces and moments which were credited to the design and position of the superelastic NiTi element as affirmed by Sander, ${ }^{(12)}$ while the stiffness of St.St. led the QH to exert excessive forces and moments ${ }^{(13,14)}$.

Effective buccal tipping aroused in the maxillary first molars for all groups. There was no statistically significant difference between NPE2 and CPB expanders which demonstrated the lowest means. QH expander showed the statistically significantly highest mean, table (1) and fig (16-c). That was the result of higher forces and moments in $\mathrm{QH}$ group. Meanwhile the lower force and moments of $\mathrm{CPB}$ was attributed to the design and position of the super elastic NiTi element. Those results are contemporaneous with those found by Marzban and Nanda, ${ }^{(10)}$ Ciambotti et al, ${ }^{(11)}$ and Sander et. al. ${ }^{(12)}$

Hicks ${ }^{(15)}$ and Cotton ${ }^{(16)}$ reported $2^{\circ}$ to $24^{\circ}$ of buccal molar tipping with use of slow expansion appliances. Marzban and Nanda, ${ }^{(10)}$ and Ciambotti et al, ${ }^{(11)}$ determined the different amounts of buccal molar tipping with the use of NPE. Herold ${ }^{(17)}$ found that minimal buccal tipping of the molars occurred with RPE and that more buccal tipping was observed with slow-expansion. Many investigators stated that with any expansion procedure, overexpansion was necessary to compensate for the tendency of the posterior teeth to return to their pretreatment axial inclination. ${ }^{(15,18,19)}$ 
Egyptian

Orthodontic Journal

\section{CONCLUSIONS}

- One of the imperative outcomes exclusive to this study was the development of a new method for 3D imaging of dental casts other than $\mathrm{CT}$ and laser scanning.

- The reliability of generating 3D dental images using photographic imaging of the dental casts for 3D tooth movement analysis has a great research potential in orthodontics because of its ability to yield accurate and reproducible data.

- An accurate and comprehensive description of the orthodontic tooth movements in various clinical situations is best assessed three dimensionally.

- The NPE2, CPB, and QH expanders are capable of expanding the maxillary dentition and alveolar process and are equally capable of correcting posterior cross bite.

\section{ACKNOWLEDGMENTS}

The authors wish to thank Dr. Ahmed Abd El Hameed Lecturer of computer science in Computer Science department, El Shorouk Academy, for his valuable assistance in the construction of the computer software.

\section{REFERENCES}

1. Kuroda T, Motohashi N, Tominanga R, Iwata K. Three-dimensional dental cast analyzing system using laser scanning. Am J Orthod Dentofacial Orthop. 1996; 110:365-369.

2. Motohashi N, Kuroda T. A 3D computer-aided design system applied to diagnosis and treatment planning in orthodontics and orthognathic surgery. Eur J Orthod. 1999; 21:263-274.

3. Papadopoulos MA, Christou PK, Athanasiou AE, Boettcher P, Zeilhofer HF, Sader R, Papadopulos NA. Three-dimensional craniofacial reconstruction imaging. Oral Surg Oral Med Oral Pathol Oral Radiol Endod. 2002; 93:382-393.

4. Hirogaki Y, Sohmura T, Satoh H, Takahashi J, Takada K. Complete 3-D reconstruction of dental cast shape using perceptual grouping. IEEE Trans Med Imaging. 2001; 20:1093-1101

5. Almeida MA, Phillips C, Kula K, Tulloch C. Stability of the palatal rugae as landmarks for analysis of dental casts. Angle Orthod. 1995; 65:43-48.

6. Virgilio F. Ferrario, Giovanna Garattini, Anna Colombo, Vittorio Filippi, Silvio, Pozzoli and Chiarella Sforza. Quantitative effects of a nickel-titanium palatal expander on skeletal and dental structures in the primary and mixed dentition: Eur J Orthod.2003; 25:401-410. 
7. Nanci L. Oliverira, Adriana C. Da Silveira, Budi Kusnoto, and Grace Viana: Three-dimensional assessment of morphologic changes of the maxilla:A comparison of 2 kinds of palatal expanders. Am J Orthod. 2004; 126:354-62.

8. Podesser B, Williams S, Crismani AG, Bantleon HP Evaluation of the effects of rapid maxillary expansion in growing children using computer tomography scanning: a pilot study. Eur J Orthod. 2007; 29(1):37-44.

9. Marini I, Bonetti GA, Achilli V, Salemi G. A photogrammetric technique for the analysis of palatal three-dimensional changes during rapid maxillary expansion. Eur J Orthod. 2007; 29(1):26-30.

10. Marzban R, Nanda R. Slow maxillary expansion with nickel titanium. J Clin Orthod. 1999; 33:431-441.

11. Ciambotti C, Ngan P, Durkee M, Kohli K, Kim HA comparison of dental and dentoalveolar changes between rapid palatal expansion and nickeltitanium palatal expansion appliances. Am J Orthod Dentofacial Orthop.2001; 119:11-20.

12. Sander C., Wichelhaus A., Franz S. Development and biomechanical investigation of a new compound palatal arch. J Orofac Orthop. 2004; 65:104-22.

13. W L. Wilson, R C. Wilson, D Modular 3D Lingual Appliances Part 1 Quad Helix. J. Clin. Orthod. 1983; 17:761 -766.

14. Donohue VE, Marshman LA, Winchester LJ. A clinical comparison of the quad helix appliance and the nickel titanium (tandem loop) palatal expander: a preliminary, prospective investigation. Eur J Orthod. 2004; 26(4):411-20.

15. Hicks, E. P.: Slow maxillary expansion: A clinical study of the skeletal versus dental response to low-magnitude force, Am. J. Orthod Dentofacial Orthop.1978; 73: 121- 141.

16. Cotton LA. Slow maxillary expansion: skeletal versus dental response to low magnitude force in Macaca Mulatta. Am J Orthod Dentofacial Orthop. $1978 ; 73: 1-22$.

17. Herold JS. Maxillary expansion: a retrospective study of three methods of expansion and their long term sequelae. Br J Orthod. 1989; 16:195-200.

18. Isaacson RJ, Murphy TD. Some effects of rapid maxillary expansion in cleft lip and palate patients. Angle Orthod. 1964; 34:143-154.

19. Storey E. The nature of tooth movement. Am J Orthod. Dentofacial Orthop. $1973 ; 63: 292-314$ 